\title{
Des données trop séduisantes
}

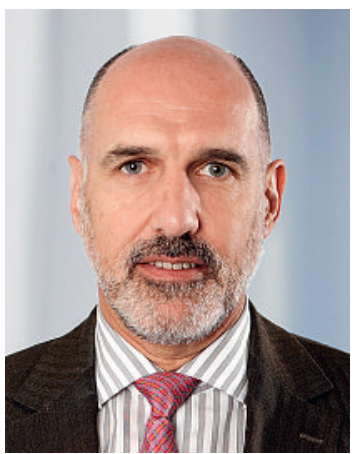

En octobre, le Parlement a rejeté les «Mesures pour endiguer l'évolution des coûts», et donc également l'article qui exigeait la transmission systématique des diagnostics aux assureurs. Par plusieurs courriers aux Parlementaires et beaucoup de contacts personnels, la FMH, tout comme H+, s'était opposée à plusieurs reprises à cette disposition qui signait la fin du secret médical.

Toutefois, malgré le rejet de ce texte, l'appétit pour davantage de données n'a pas changé: le traitement et la transmission électroniques semblent même l'exciter encore plus, selon l'idée erronée que davantage de données signifierait davantage de connaissances, et donc davantage de pouvoir.

La FMH demande depuis longtemps la mise en œuvre de la cybersanté pour soutenir et optimiser la prise en charge des patients; la cybersanté est un instrument important qui permet de mieux gérer les processus thérapeutiques, de les rendre plus sûrs, et qui permet aussi la réalisation de nouveaux concepts thérapeutiques intégrés.

\section{SwissDRG: le contrôle du codage doit être confié à des réviseurs professionnels externes}

Mais nous le vérifions chaque jour: pour qu'un traitement réussisse, les patients doivent pouvoir nous faire entièrement confiance et savoir que ce qu'ils nous disent restera entre nous. Ce n'est ni par la contrainte de fournir une documentation électronique ni par des primes d'assurance plus basses qu'on pourra imposer la confiance qui nous lie à nos patients. Elle n'existera que si le médecin protège de façon adéquate les informations qui lui sont confiées.

Or bien souvent, l'appétit des assureurs pour les données n'est pas compatible avec cette confiance et à l'avenir, nous devrons encore accroître notre vigilance pour que le secret médical ne devienne pas une coquille vide.

Dans le domaine stationnaire, il n'est pas nécessaire que des données détaillées figurent sur chaque facture pour contrôler les divers hôpitaux. Les statistiques médicales de l'Office fédéral de la statistique sont la preuve qu'on peut tout à fait établir les profils des hôpitaux avec des données anonymisées.

La FMH aussi soutient un contrôle approprié du codage des factures DRG. Mais comme ce contrôle ne peut avoir lieu que sur la base du dossier médical, seules des révisions externes et professionnelles peuvent être envisagées: externes parce qu'elles seront réalisées par des réviseurs indépendants, et professionnelles parce que ces derniers auront acquis les qualifications requises. Ces réviseurs externes devront être mandatés d'un commun accord par les partenaires tarifaires. Il est évidemment hors de question de permettre l'accès aux dossiers médicaux aux employés de chaque assureur isolément.

\section{Les indications sur les factures Spitex et des EMS violent également la sphère privée et le droit du patient à l'autodétermination}

Dans la médecine ambulatoire également, il importe de clairement séparer moyens et objectifs! Les assureurs veulent toujours davantage contrôler le traitement proprement dit parallèlement au contrôle des coûts qu'il leur incombe d'effectuer. Leurs tentatives d'exiger l'indication du diagnostic de tous les patients sur chaque facture vont dans cette direction. L'indication sur les factures des diagnostics CIM détaillés, telle qu'elle avait été prévue dans le paquet de mesures urgentes, aurait entraîné des coûts supplémentaires et d'importantes contraintes administratives. Par ailleurs, il est souvent difficile dans le domaine ambulatoire de poser des diagnostics atteignant un tel degré de détail. Mais surtout cela aurait de facto abrogé le secret médical.

Une facture doit passer entre les mains des différents employés d'un assureur et elle est ensuite conservée durant des années; elle est donc accessible à un grand nombre de personnes inconnues. Selon la situation personnelle et sociale de l'intéressé, la facture doit être transmise également à des membres de sa famille, à l'aide sociale, aux autorités tutélaires ou fiscales. Ces autorités n'ont pas davantage le droit de tout savoir, de connaître les infections dont est atteinte une personne ou le diagnostic sur la base duquel elle consulte un psychiatre. A noter que la sphère privée et le droit du patient à l'autodétermination en matière d'information sont susceptibles d'être violés non seulement par les factures des médecins, mais également par les factures Spitex ou celles des établissements médico-sociaux, par exemple en cas d'indication de démence ou d'incontinence.

Par conséquent, la FMH devra, à l'avenir également, contenir dans des limites raisonnables l'appétit des assureurs en matière de données. Les principes fondamentaux de la médecine, en l'occurrence, le secret médical, ne sont pas négociables!

Dr Jacques de Haller, président de la FMH 